\title{
Exploring the Geometry of the Space of Shells
}

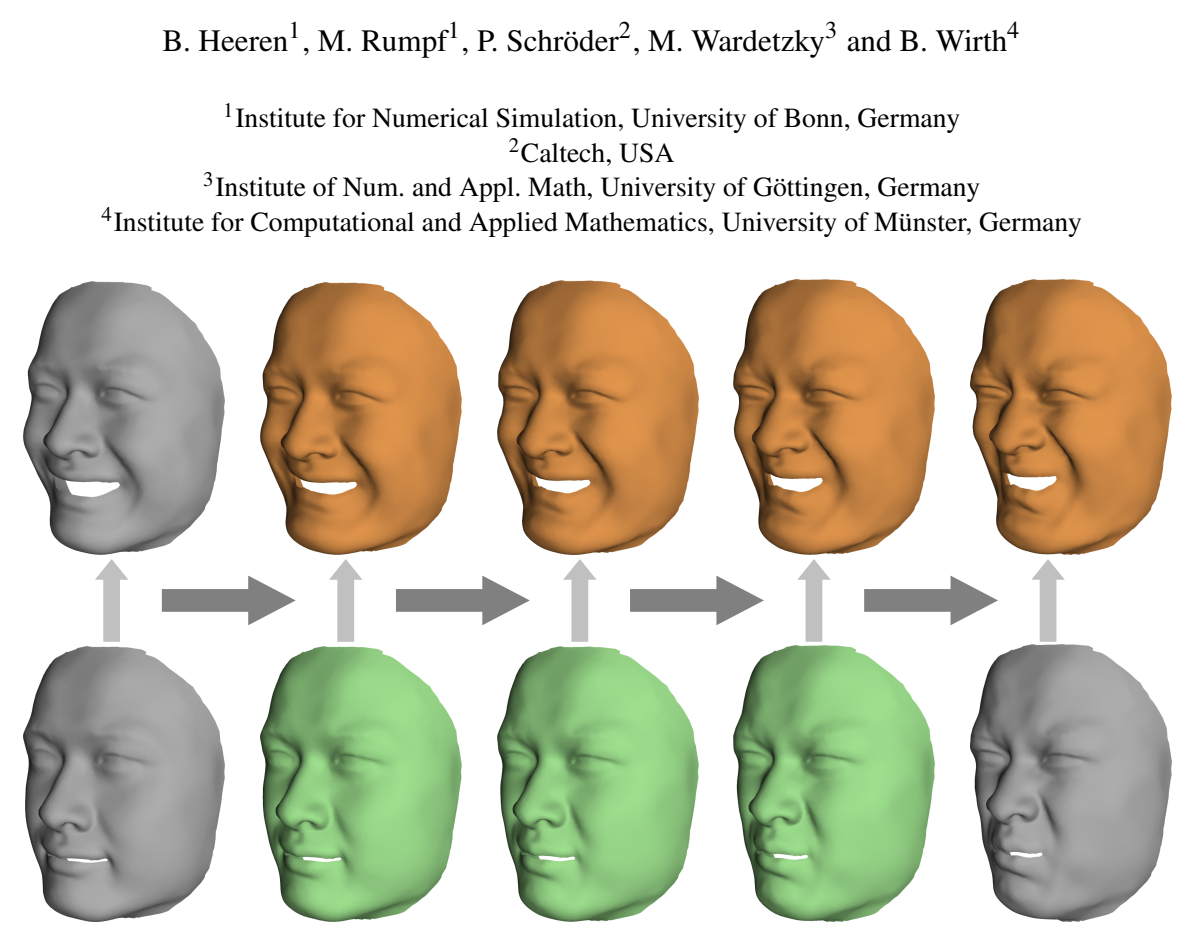

Figure 1: With geodesic paths we can parallel transport the difference between a smiling (upper left) and a neutral face (lower left) along a path (bottom row) towards a disgusted face (bottom right), resulting in a smile with a frown (upper right).

\begin{abstract}
We prove both in the smooth and discrete setting that the Hessian of an elastic deformation energy results in a proper Riemannian metric on the space of shells (modulo rigid body motions). Based on this foundation we develop a time- and space-discrete geodesic calculus. In particular we show how to shoot geodesics with prescribed initial data, and we give a construction for parallel transport in shell space. This enables, for example, natural extrapolation of paths in shell space and transfer of large nonlinear deformations from one shell to another with applications in animation, geometric, and physical modeling. Finally, we examine some aspects of curvature on shell space.
\end{abstract}

Categories and Subject Descriptors (according to ACM CCS): I.3.5 [Computer Graphics]: Computational geometry and object modeling-Physically based modeling

\section{Introduction}

Elastic energies play a pivotal role when deforming, animating, or simulating geometries. Continuously changing the form of a given shape corresponds to a continuous path in shape space. Studying these paths offers a way to explore shape space, both locally and globally. Geometrically, such an exploration relates to the search for shortest paths, or geodesics, which require a Riemannian metric for distance measurements. Physically, the exploration relies on a notion of distance between shapes given by the energy dissipated while deforming one shape into another. Here we combine geometry and physics by showing that the Hessians of a wide 
class of commonly used elastic energies for thin shells give rise to Riemannian metrics on shell space. In hindsight, this justifies the notion of geodesic paths in shell space introduced in [HRWW12].

With this foundation we can build a geodesic calculus, for example, a notion of parallel transport in shape space. That is, we can move a vector field along geodesic paths and in this way transfer, for example, large nonlinear deformations from one shape to another (see Fig. 1). In the time- and space-discrete setting this amounts to defining

- a discrete notion of the logarithm, mapping shortest paths between two shapes into an initial velocity vector which "shoots" to the target shape, and

- a discrete notion of the exponential, mapping an initial shooting direction into a particular shape via a shortest path.

Practically speaking, the final algorithm consists of sequences of elementary steps: finding a third shape as the minimizer of an action involving three shapes, two of which are given. Because each of these steps represents a variational problem, our approach is linked to the variational discretization of Hamiltonian systems in mechanics, which helps ensure computational robustness.

Aside from practical applications in geometric modeling, such as animation and deformation transfer, these tools also allow us to explore the local and global geometry of shell space, which are little understood so far. We provide a first glimpse in this direction via examples of Riemannian triangles in shell space as well as examples of a particular consequence of space curvature in Riemannian geometry known as holonomy.

\section{Related work.}

Here we briefly review some of the relevant concepts and paradigms required in our geometric treatment of shell space.

Elastic energy of shells. The importance of efficient simulations and deformations of thin plates and shells has long been known to the graphics community [TPBF87]. Various researchers in graphics have formulated and discretized separately the membrane and bending modes of deformation of Kirchhoff-Love shells. The treatment of the membrane energy for triangulated meshes follows the widely studied models of elasticity in the Finite Element community [ZT00, Hug87]. In contrast, the geometrically nonlinear treatment of bending energy, which accounts for change of curvature, requires tools from (discrete) differential geometry. In graphics, popular approaches for the treatment of bending energy include discrete thin shells [GHDS03] and a discrete shape operator based on edge normals [GGRZ06]. Alternative representations of metric and bending data in the form of edge lengths and dihedral angles have been used by Winkler et al. [WDAH10] in the context of cascadic interpolation, while PriMo [BPGK06] introduced a non-linear model for the deformation of thick shells (rigid prisms coupled by non-linear springs). It is also possible to include learning into physical simulation for shape deformation as investigated by Fröhlich and Botsch [FB11]. For an overview of linear deformation models (as opposed to geometrically nonlinear ones that we use here) we refer to [BS08].

Relative to all these previous approaches ours is characterized by taking a differential geometric point of view. Deformation energy densities are functions of the relative first and second fundamental forms, incorporating geometric nonlinearities while being agnostic to the constitutive model used.

Hessian of elastic energy. Physical simulation models can be expressed in different bases. One attractive choice is the so called modal basis given by the eigenvectors of the energy Hessian. Noting that small eigenvalues correspond to low stiffness, the corresponding eigenvectors parameterize physically preferred deformation modes. This was exploited recently by Hildebrandt et al. [HSTP11, TSSH13], who used this basis for the intuitive modeling of surfaces and acceleration of physical simulations with a linearized vibration model and damping.

In our case, small eigenvalues of the energy Hessian resemble low frequency oscillations, i.e., they produce the least energy dissipation for a given magnitude of motion. We utilize this fact when doing geodesic extrapolation.

Geometry of spaces of shapes. From a more global perspective, researchers in vision have studied shapes as points in shape space. Studying shape space from the point of view of Riemannian geometry enables transfer of important concepts from classical geometry to these (usually) infinitedimensional spaces. Examples with a fully developed geometric theory include spaces of planar curves with curvature based metrics [MM06], elasticity based metrics [SJJK06], or Sobolev-type metrics [SYM07]. Geodesic paths between shapes have been approximated via the minimization of discretized path length [SCC06] or path energy [FJSY09, WBRS11]. This Riemannian perspective has had a large impact ranging from shape morphing and modeling, see, e.g., [KMP07], to shape statistics, see, e.g., [FLPJ04], and computational anatomy [BMTY02]. A central concept in this setup is the flow of diffeomorphisms to define distances and geodesics. See [You10] for a comprehensive exposition.

Most relevant for us is the work of Kilian et al. who investigated the (finite dimensional) space of triangulated surfaces and considered geodesics between such meshes [KMP07], with respect to a Riemannian metric measuring the stretching of triangle edges. While this metric is invariant to rigid body motions, the lack of a bending term leaves a nontrivial kernel of the metric tensor, including all isometric deformations of the triangular mesh. To avoid the resulting unphysical wrinkling effects, a supplementary (non physical) regularization was incorporated by Killian and co-workers. Instead one may use the regularizing effect of bending energy - and stay entirely in a physical simulation 
framework-as demonstrated in [HRWW12]. Other types of shape spaces where explored by Kurtek et al. [KKG*12], who studied geodesic paths between surfaces parametrized over the unit sphere, using local changes of the area element as a Riemannian metric; Bauer et al. [BHM11] investigated geodesic paths on the space of surfaces described by embeddings or immersions of a given manifold using as Riemannian metric a quadratic form corresponding to a higher order elliptic operator; finally Jin et al. [JZLG09] studied Teichmüller space as a finite dimensional shape manifold, where shapes are classes of conformally equivalent surfaces.

Our method falls into the category of shape space based approaches and we show rigorously that the energy Hessian of a simple class of stretching/bending models does indeed provide a proper Riemannian metric.

Link to variational integrators in mechanics. For Hamiltonian mechanical systems, variational and structure preserving time discretization is by now a classic field [HLW06]. The discretization of the Lagrangian in mechanics [LMOW04] corresponds to our notion of a timediscrete path energy. From the discrete Lagrangian, by using a discrete Hamilton's principle, one arrives at our EulerLagrange equation of discrete geodesics. The associated discrete symplectic time steps exhibit the same structure as the discrete exponential shooting proposed here, giving it many of the numerical advantages associated with such methods.

\section{Riemannian structure of the space of shells}

Here we outline the elastic energies that we consider in our exposition, both for the case of smooth and discrete surfaces, and we show that energy Hessians give rise to Riemannian structures.

Smooth thin shells. Shells are thin, curved material sheets, i.e., three-dimensional objects $s \delta$ of thickness $\delta>0$. We denote their midsurface by $s$. A deformation $\phi^{\delta}: s^{\delta} \rightarrow \mathbb{R}^{3}$ of the physical material $s^{\delta}$ maps each point $x \in s^{\delta}$ onto a new position $\phi^{\delta}(x)$. By $\mathcal{W}^{\delta}\left[\phi^{\delta}\right]$ we denote the stored (in general nonlinear) elastic energy of the deformation $\phi^{\delta}$. It is wellknown (see, e.g., the exposition in [HRWW12]) that up to higher order terms in $\delta$ this $3 \mathrm{D}$ energy reduces to a rescaled energy $\mathcal{W}[\phi]$ on the deformation of the midsurface $s$.

For a homogeneous, isotropic, elastic material, the (rescaled) deformation energy $\mathcal{W}[\phi]$ of the elastic deformation of the midsurface $s$ can be captured by membrane and bending contributions, or the changes of first (i.e., metric) and second (i.e., curvature) fundamental forms. Accordingly, we define

$$
Q_{\mathrm{mem}}[\phi]=B_{\phi}^{\mathrm{mem}}-B_{\mathbb{1}}^{\mathrm{mem}} \quad \text { and } \quad Q_{\text {bend }}[\phi]=B_{\phi}^{\text {bend }}-B_{\mathbb{1}}^{\text {bend }}
$$

with the symmetric operators $B^{\text {mem }}$ and $B^{\text {bend }}$ given by

$$
\begin{aligned}
& \mathrm{I}_{s}\left(B_{\phi}^{\text {mem }} v, w\right)=\mathrm{I}_{\phi(s)}(\mathrm{d} \phi(v), \mathrm{d} \phi(w)), \\
& \mathrm{I}_{s}\left(B_{\phi}^{\text {bend }} v, w\right)=\mathrm{II}_{\phi(s)}(\mathrm{d} \phi(v), \mathrm{d} \phi(w)),
\end{aligned}
$$

Here, $\mathrm{I}_{s}, \mathrm{II}_{s}$, and $v, w$ denote the first and second fundamental form as well as two tangent vectors of $s$, all at a position $x \in s$. These equations uniquely define $B^{\mathrm{mem}}$ and $B^{\text {bend }}$ as they have to hold for all $v$ and $w$. The operators $B^{\mathrm{mem}}$ and $B^{\text {bend }}$ are the linear operators that correspond to the quadratic first and second fundamental forms, respectively.

The total elastic energy is then given by

$\mathcal{W}[\phi]=\delta \int_{S} W_{\text {mem }}\left(Q_{\text {mem }}[\phi]\right) \mathrm{d} x+\delta^{3} \int_{S} W_{\text {bend }}\left(Q_{\text {bend }}[\phi]\right) \mathrm{d} x$,

where the non-negative energy densities $W_{\text {mem }}, W_{\text {bend }}$ act on the symmetric linear rank two operators $Q_{\text {mem }}$ and $Q_{\text {bend }}$. A prominent example for these densities is the squared Frobenius norm. For $W_{\text {mem }}$ and $W_{\text {bend }}$ we require that (i) $W(0)=0$, (ii) $D W=0$ at the zero matrix, and (iii) $D^{2} W$ is positive definite at the zero matrix. These requirements correspond to the fact that if the shell is in a stress free configuration, then the deformation identity $\mathbb{1}$ is a minimizer of $\mathcal{W}$ and thus (i) $\mathcal{W}[\mathbb{1}]=0$ and (ii) $d \mathcal{W}[\mathbb{1}]=0$. Additionally, we assume (iii) that the energy is strictly convex (modulo rigid body motions) in a neighborhood of a minimizer. These assumptions capture most thin elastic materials [Cia00].

Let $\mathcal{S}$ denote the space of smooth shells. Our first main result is:

Thm. 1 (Non-degeneracy of smooth Hessian) For $v$ a tangent vector field to $\mathcal{S}$ at some smooth $s \in \mathcal{S}$, $\operatorname{Hess}(\mathcal{W})(v, v)=0$ if and only if $v$ induces an infinitesimal rigid motion. Consequently, $g_{s}(v, w)=\frac{1}{2} \operatorname{Hess}(\mathcal{W})(v, w)$ is indeed a Riemannian metric on the space of smooth shells modulo infinitesimal rigid body motions.

We defer the proof to the appendix.

Discussion. Note that in general the Hessian Hess $(f)=$ $\nabla(d f)$ of a function $f$ depends on the choice of a Riemannian metric through the covariant derivative $\nabla$. Thus in general it is meaningless to speak of Hessians giving rise to a Riemannian metric without presuming such a metric to begin with. However, at a critical point of a function $f$ (i.e., a point where $d f=0)$, Hess $(f)$ is independent of the choice of metric [Mil63]. This is our setting since a given shell is the minimizer of its elastic energy.

Discrete thin shells. We identify a triangulated shell or surface $\mathbf{s}$ with a vector $\mathbf{s} \in \mathbb{R}^{3 m}$, where $m$ denotes the number of vertices. We follow the smooth setting for our exposition in the discrete case.

A deformation of a triangulated shell can be identified with a mapping $\phi: \mathcal{V} \rightarrow \mathbb{R}^{3}$, where $\mathcal{V}$ denotes the set of vertices. If the deformation $\phi$, acting on vertices, is interpolated piecewise linearly over triangles, then the membrane part can be treated identically to the smooth case. For the bending part, which requires second derivatives of surface positions in the smooth case, we resort to (a variant of) the widely used thin shell energy in the discrete case, where bending is 
quantified by changes of dihedral angles. In both cases the discrete energy densities $W_{\text {mem }}$ and $W_{\text {bend }}$ can be chosen identically to their smooth counterparts.

The discrete version of the elastic deformation energy $\mathcal{W}$ reads

$\mathbf{W}[\phi]=\delta \sum_{t \in \mathcal{T}} W_{\text {mem }}\left(Q_{\text {mem }}^{t}[\phi]\right) A_{t}+\delta^{3} \sum_{e \in \mathcal{E}} W_{\text {bend }}\left(Q_{\text {bend }}^{e}[\phi]\right) A_{e}$,

where $\mathcal{T}$ and $\mathcal{E}$ denotes the set of triangles and edges, respectively, $A_{t}$ denotes the triangle area of the undeformed triangle $t$, and $A_{e}$ is the area associated with an undeformed edge $e$. The latter is given by $A_{e}=|e| h_{e}=\frac{1}{3}\left(A_{t_{1}}+A_{t_{2}}\right)$, where $h_{e}$ is a third of the average of the two heights of the two triangles $t_{i}$ incident to $e$. Finally,

$$
Q_{\mathrm{mem}}^{t}[\phi]=B_{\phi, t}^{\mathrm{mem}}-B_{\mathbb{1}, t}^{\mathrm{mem}} \quad \text { and } \quad Q_{\mathrm{bend}}^{e}[\phi]=B_{\phi, e}^{\mathrm{bend}}-B_{\mathbb{1}, e}^{\text {bend }},
$$

where $B_{\phi, t}^{\mathrm{mem}}$ and $B_{\phi, e}^{\mathrm{bend}}$, respectively, are given by

$$
\begin{aligned}
B_{\phi, t}^{\mathrm{mem}} & =\frac{1}{8 A_{t}^{2}} \sum_{i=0}^{2}\left(\mathrm{I}_{j}^{\phi}+\mathrm{I}_{k}^{\phi}-\mathrm{I}_{i}^{\phi}\right)\left(* e_{i}\right) \otimes\left(* e_{i}\right), \\
B_{\phi, e}^{\text {bend }} & =\frac{\theta_{\phi(e)}}{h_{e}} \frac{(* e) \otimes(* e)}{|e|^{2}} .
\end{aligned}
$$

Here, the indices $i, j, k \in\{0,1,2\}$ refer to the edges of $t$, where $j=i+1(\bmod 3)$ and $k=i+2(\bmod 3), \otimes$ denotes the outer product, and $* e_{i}$ is the undeformed edge $e_{i}$ rotated clockwise by $\pi / 2$ in the plane of $t$. Similarly $* e$ is the undeformed edge $e$ rotated clockwise by $\pi / 2$ in the plane of one (arbitrary) of the triangles incident to $e, \theta_{\phi(e)}$ is the dihedral angle at the deformed edge $\phi(e)$, and $\mathrm{I}_{i}^{\phi}$ is defined as $\mathrm{I}_{i}^{\phi}=\left\|\phi\left(e_{i}\right)\right\|^{2}$ for the deformed $i^{\text {th }}$ edge $\phi\left(e_{i}\right)$.

Simplified expressions for discrete elastic energy. We chose the above representation of discrete elastic energies since it enables reuse of the smooth energy densities $W_{\text {mem }}$ and $W_{\text {bend. }}$ When $W_{\text {bend }}$ is given by the squared Frobenius norm (our setting), the representation can be simplified to the widely used discrete shells energy

$$
\mathbf{W}[\phi]_{\text {bend }}=\delta^{3} \sum_{e \in \mathcal{E}} \frac{|e|}{h_{e}}\left(\theta_{e}-\theta_{\phi(e)}\right)^{2} .
$$

For $W_{\text {mem }}$ we use the nonlinear energy density given in equation (8) in [HRWW12].

Let $\mathbf{S}$ denote the space of triangulated shells. Our second main result is:

Thm. 2 (Non-degeneracy of discrete Hessian) For $v \in \mathbb{R}^{3 m}$ a tangent vector to $\mathbf{S}$ at some $\mathbf{s} \in \mathbf{S}$, $\operatorname{Hess}(\mathbf{W})(\mathbf{v}, \mathbf{v})=0$ if and only if $\mathbf{v}$ induces an infinitesimal rigid motion. Consequently, $g_{\mathbf{s}}(\mathbf{v}, \mathbf{w})=\frac{1}{2} \operatorname{Hess}(\mathbf{W})(\mathbf{v}, \mathbf{w})$ is indeed a Riemannian metric on the space of discrete shells modulo rigid body motions.

Proof Suppose that Hess $(\mathbf{W})(\mathbf{v}, \mathbf{v})=0$ for some vector field $\mathbf{v}$ sitting at the vertices. Then $\mathbf{v}$ is both in the kernel of the Hessian of discrete membrane energy and the Hessian of discrete bending energy since both are positive semi-definite operators. Consider two adjacent triangles $t_{1}$ and $t_{2}$. After subtracting global translations induced by $\mathbf{v}$, for $\mathbf{v}$ to be in the kernel of the Hessian of membrane energy implies that $\mathbf{v}$ induces an infinitesimal rotation of $t_{1}$ since the edge lengths of $t_{1}$ must not change. The same holds for $t_{2}$. Since $\mathbf{v}$ is also in the kernel of the Hessian of bending energy, it follows that $\mathbf{v}$ must not induce a change of the dihedral angle between $t_{1}$ and $t_{2}$. Hence $\mathbf{v}$ induces a single infinitesimal rotation of the hinge $t_{1} \cup t_{2}$. Iterating this argument over the entire mesh proves the claim.

Physical interpretation We have established a Riemannian metric both on the space of continuous and discrete shells. Physically, our approach corresponds to thin shells that dissipate energy when being deformed. A widespread model of dissipation in mechanics [Hug87,ZT00] is Rayleigh damping. By Rayleigh's paradigm [Str45] one derives models for viscous dissipation from elastic energies replacing elastic strains by strain rates. Consider a 1-parameter family $\left(\phi_{t}\right)$ of diffeomorphisms of $\mathbb{R}^{3}$ with $\phi_{0}=\mathbb{1}$ and let $v=\dot{\phi}(0)$ denote the Eulerian velocity at time $t=0$. Replacing strains $\phi_{t}-\mathbb{1}$ for small times $t$ by strain rates $v$ we obtain (up to rescaling of time and higher oder spatial terms) the rate of viscous dissipation $g_{s}(v, v)=\frac{1}{2} \operatorname{Hess} \mathcal{W}[\mathbb{1}](v, v)$ for a given shell $s$. In particular, geodesics in shell space correspond to paths of least energy dissipation. Note the difference between elastic energy and viscous dissipation: elastic energies are independent of the deformation path, whereas dissipation is not. In elastic dynamics energy is preserved and is given as a sum of kinetic and potential (stored elastic) energy. In the case of viscous dissipation dynamics, energy is dissipated (converted) into heat due to internal friction.

\section{Discrete geodesic calculus}

Having established that geodesic paths, with respect to a Riemannian metric arising from the Hessian of an elastic energy, are well-defined, we now use this Riemannian metric to develop a discrete geodesic calculus on shape space. In the smooth setting, where we consider a continuous family $(\phi(t, \cdot))_{t \in[0,1]}$ of deformations $x \mapsto \phi(t, x)$ and an induced path $(s(t))_{t \in[0,1]}$ in the space of shells with $s(t)=\phi\left(t, s_{A}\right)$ and $\phi(0, \cdot)=\mathbb{1}$ for a given shell $s_{A}$, one obtains the path energy

$$
\mathcal{E}\left[(s(t))_{t \in[0,1]}\right]=\int_{0}^{1} g_{s(t)}(v(t), v(t)) \mathrm{d} t
$$

with $v=\dot{\phi} \circ \phi^{-1}$ the Eulerian motion field. Minimizers of the path energy for fixed end points $s(0)$ and $s(1)$ are shortest paths which are also unit speed parameterized.

In the discrete setting this path energy integral is replaced with a sum of elastic deformation energies between successive intermediate shapes as suggested in [HRWW12]

$$
E\left[s_{0}, \ldots, s_{K}\right]=\frac{1}{\tau} \sum_{k=1}^{K} \mathcal{W}\left[s_{k-1}, s_{k}\right]
$$


where $\left(s_{0}, \ldots, s_{K}\right)$ is a time-discrete path with time step size $\tau=\frac{1}{K}$ and $\mathcal{W}[s, \tilde{s}]$ is the elastic energy for a mapping $\phi$ of $s$ onto $\tilde{s}$. In analogy with the smooth setting, we call minimizers for fixed end shapes $s_{0}$ and $s_{K}$ time discrete geodesics.

When the shell is also discretized in space we get a fully discrete path energy $\mathbf{E}\left[\mathbf{s}_{0}, \ldots, \mathbf{s}_{K}\right]$ by replacing $\mathcal{W}\left[s_{k-1}, s_{k}\right]$ with its discrete counterpart $\mathbf{W}\left[\mathbf{s}_{k-1}, \mathbf{s}_{k}\right]$. Using a proper discretization of an underlying smooth energy in space and time distinguishes our approach from others such as [FB11, KMP07]. Consequently our path energy is much less dependent on the underlying mesh and consistent under refinement.

Geodesic interpolation and extrapolation. Consider the case $K=2$ with shapes $\mathbf{s}_{0}, \mathbf{s}_{1}$, and $\mathbf{s}_{2}$. If $\mathbf{s}_{0}$ and $\mathbf{s}_{2}$ are given, the interpolated geodesic midpoint $\mathbf{s}_{1}$ can be found as the minimizer of the discrete path energy, $\mathbf{s}_{1}=$ $\operatorname{argmin}_{\mathbf{s}} \mathbf{E}\left[\mathbf{s}_{0}, \mathbf{s}, \mathbf{s}_{2}\right]$, i.e., as the zero $\mathbf{s}_{1}$ of the corresponding Euler-Lagrange equation

$$
0=\partial_{2} \mathbf{E}\left[\mathbf{s}_{0}, \mathbf{s}_{1}, \mathbf{s}_{2}\right]=\frac{1}{\tau}\left(\partial_{2} \mathbf{W}\left[\mathbf{s}_{0}, \mathbf{s}_{1}\right]+\partial_{1} \mathbf{W}\left[\mathbf{s}_{1}, \mathbf{s}_{2}\right]\right)
$$

(where $\partial_{i}$ denotes the derivative with respect to the $i$ th argument). If instead a starting point $\mathbf{s}_{0}$ and a direction $\mathbf{s}_{1}-\mathbf{s}_{0}$ are given, the next shape $\mathbf{s}_{2}$ is found by geodesic extrapolation, i.e., this time we have to find $\mathbf{s}_{2}$ such that the same equation, $0=\partial_{2} \mathbf{E}\left[\mathbf{s}_{0}, \mathbf{s}_{1}, \mathbf{s}_{2}\right]$, holds with $\mathbf{s}_{0}, \mathbf{s}_{1}$ fixed.

Now consider $K>2$ (see, e.g., Fig. 4). For given end shapes $\mathbf{s}_{0}$ and $\mathbf{s}_{K}$, the interpolating shapes are found again by minimizing $E\left[\mathbf{s}_{0}, \mathbf{s}_{1}, \ldots, \mathbf{s}_{K-1}, \mathbf{s}_{K}\right]$. For given starting point $\mathbf{s}_{0}$ and direction $\mathbf{s}_{1}-\mathbf{s}_{0}$, the extrapolated shapes $\mathbf{s}_{2}, \ldots, \mathbf{s}_{K}$ are obtained step by step, simply applying the above threepoint case iteratively. Note that the interpolated geodesic $\mathbf{s}_{0}, \ldots, \mathbf{s}_{K}$ and the geodesic extrapolation from $\mathbf{s}_{0}$ in direction $\mathbf{s}_{1}-\mathbf{s}_{0}$ coincide because the defining Euler-Lagrange equation is the same and the minimizer of the variational problem is unique. Indeed, for discrete shells $\mathbf{s}_{0}, \mathbf{s}_{K}$ close to each other, the latter is guaranteed due to Theorem 2, which implies local strict convexity.

Notice how we may think of discrete geodesic interpolation as a map from a geodesic path ( $\mathbf{s}_{0}$ to $\left.\mathbf{S}_{2}\right)$ to a difference vector $\mathbf{s}_{1}-\mathbf{s}_{0}$, while discrete geodesic extrapolation can be seen as a map from a difference vector $\mathbf{s}_{1}-\mathbf{s}_{0}$ to a geodesic path connecting $\mathbf{s}_{0}$ to $\mathbf{s}_{2}$. The smooth counterparts of these operations, respectively, are the logarithm map, which maps a geodesic path to an initial velocity vector, and the exponential map which maps an initial velocity to a geodesic path.

Fig. 3 shows an application of interpolation and extrapolation (top). Furthermore, the rate of viscous dissipation is plotted to show the equidistribution of dissipation along the discrete geodesic path (bottom). Fig. 4 shows an example based on large, nonlinear deformations. Again, the equidistribution of dissipation is striking - in particular, when compared to other approaches. Fig. 2 demonstrates the robustness of the proposed time discrete geodesic extrapolation,

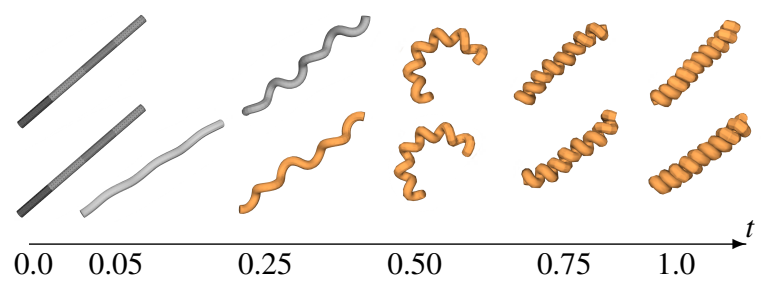

Figure 2: Shape extrapolation is applied to generate a strong twisting of a helix (see Fig. 6 in [FB11]) with $\delta=$ $\sqrt{10^{-5}}$. The two gray surfaces are taken from the computational results in [FB11] (at times 0.0 and 0.25 (top), 0.0 and 0.05 (bottom)) and used as input data here.

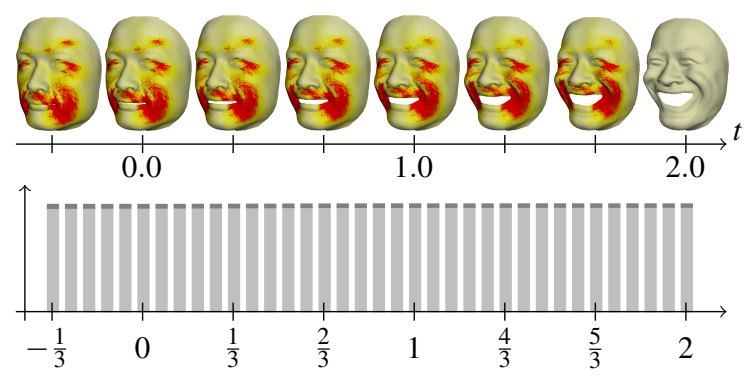

Figure 3: Top: Geodesic interpolation for $0 \leq t \leq 1$ of given shells at time 0 and 1, geodesic extrapolation for $t<0$ and $t>1$ (local dissipation rate is color coded as $0 \quad 1$; nominal shell thickness is $\delta=1)$. Bottom: The total dissipation rate (membrane contribution in light gray, bending contribution in dark gray) stays constant along the path.

both for large time steps and given large nonlinear initial variation as well as for many small time steps and small initial offset.

With both a discrete version of logarithm and exponential available we can now perform not only geodesic interpolation but also parallel transport, to which we now turn.

Parallel transport. The continuous parallel transport of a tangent vector $\mathbf{v} \in T_{\mathbf{S}(0)} \mathbf{S}$ along the path $(\mathbf{s}(t))_{t \in[0,1]}$ is the tangent vector $\mathbf{v}(1)$ resulting from the solution of $\nabla_{\dot{\mathbf{s}}(t)} \mathbf{v}(t)=0$ for $t \in[0,1]$ and initial data $\mathbf{v}(0)=\mathbf{v}$, where $\nabla_{\dot{\mathbf{s}}}$ denotes the covariant derivative based on the Levi-Civita connection. It can be approximated (still in the continuous setting) using a Riemannian (or Levi-Civita) parallelogram construction first used in gravitational physics and named Schild's ladder [EPS72, KMN00]: Given three shells $\mathbf{s}_{A}, \mathbf{s}_{B}$, and $\mathbf{s}_{C}$, we can construct a fourth shell $\mathbf{s}_{D}$ such that the geodesic paths between the four shells form a geodesic parallelogram, i.e., the geodesics $\mathbf{s}_{C} \leftrightarrow \mathbf{s}_{B}$ and $\mathbf{s}_{A} \leftrightarrow \mathbf{s}_{D}$ share the same midpoint $\mathbf{s}^{\times}$(see Fig. 5). To compute $\mathbf{s}_{D}$ we proceed as follows: (i) compute the geodesic from $\mathbf{s}_{C}$ to $\mathbf{s}_{B}$ via interpolation; (ii) take its midpoint $\mathbf{s}^{\times}$, (iii) compute the geodesic from $\mathbf{s}_{A}$ to $\mathbf{s}^{\times}$, (iv) compute $\mathbf{s}_{D}$ through extrapolation from $\mathbf{s}^{\times}$with initial direction $\mathbf{v}^{\times}$, where $\mathbf{v}^{\times}$is the final velocity 


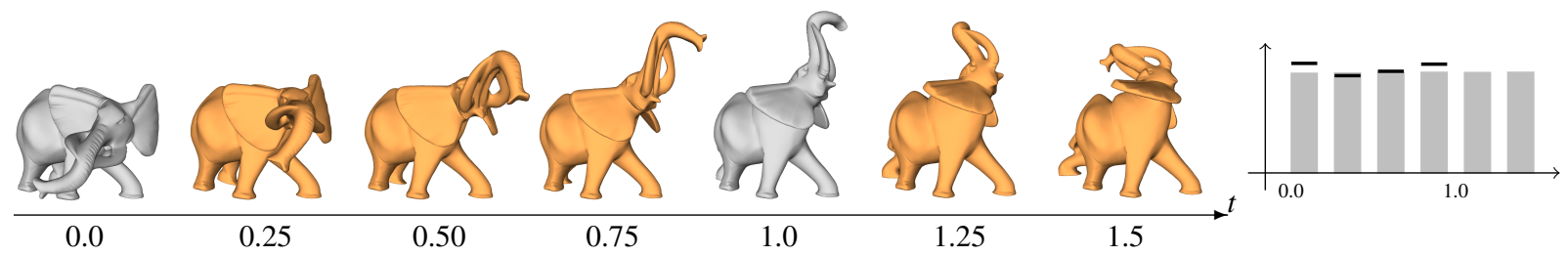

Figure 4: The two gray elephant surfaces are connected via a discrete geodesic with $K=4$ and for $\delta=\sqrt{10^{-5}}$. The geodesic is extended using geodesic extrapolation (see Fig. 11 in [WDAH10] and Fig. 5 in [FB11]). On the right the corresponding rate of viscous dissipation is plotted for the 6 time steps. Furthermore, black lines indicate the dissipation for the corresponding sequence from [WDAH10]. Edge lengths and dihedral angles deviate from linearly interpolated lengths and angles by at most $72 \%$ (resp. $29^{\circ}$ ) and by at most $2.5 \%$ (resp. $1.5^{\circ}$ ) when omitting the worst $1 \%$ edges (see Fig. 7 and 8 in [WDAH10]).

of the geodesic in step (iii) at its endpoint $\mathbf{s}^{\times}$. A sequence of $N$ such parallelograms then transports the initial velocity of $\mathbf{s}_{A} \leftrightarrow \mathbf{s}_{C}$ along the path from $\mathbf{s}_{A}$ to $\mathbf{s}_{N}$ to the initial velocity of $\mathbf{s}_{N} \leftrightarrow \mathbf{s}_{N}^{\prime}$ (see also Fig. 5).

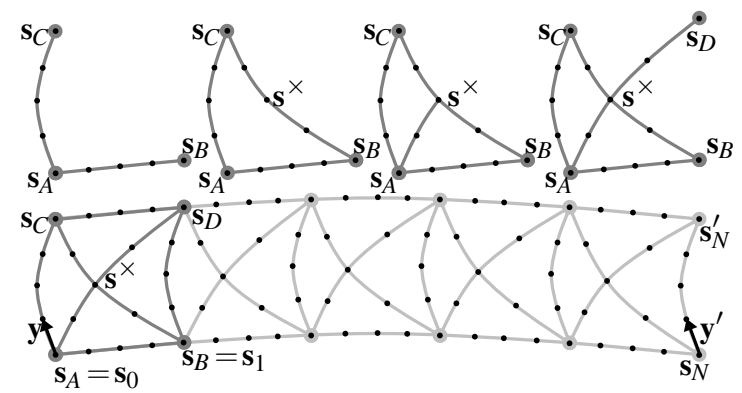

Figure 5: Top: Stepwise construction of a (discrete) geodesic parallelogram (in the discrete case $K=4$ such that each discrete geodesic consists of five shapes indicated by the black dots). Bottom: Iterative construction for the timediscrete parallel transport of $\mathbf{y}$ along $\left(\mathbf{s}_{0}, \ldots, \mathbf{s}_{N}\right)$ to $\mathbf{y}^{\prime}$.

The parallelogram construction can easily be transferred to the time-discrete setup. Effectively, to compute $\mathbf{s}_{D}$ we proceed along the same steps (i) to (iv), only replacing geodesics by discrete geodesics. Iterating this discrete geodesic parallelogram construction as in Fig. 5 (the black dots refer to discrete geodesics with $K=4$ ), we transport the pair $\left(\mathbf{s}_{A}, \mathbf{s}_{C}\right)$ along the discrete path $\left(\mathbf{s}_{0}=\mathbf{s}_{A}, \ldots, \mathbf{s}_{N}\right)$ to obtain the pair $\left(\mathbf{s}_{N}, \mathbf{s}_{N}^{\prime}\right)$. The first step of the discrete geodesic from $\mathbf{s}_{N}$ to $\mathbf{s}_{N}^{\prime}$ then is the parallel transported first step of the discrete geodesic from $\mathbf{s}_{A}$ to $\mathbf{s}_{C}$. Figure 1 uses such a concatenation of geodesic parallelograms to transport a smile along a path from a neutral to a disgusted facial expression.

Given a shell surface $\mathbf{s}$ and two variants $\mathbf{s}^{\prime}$ and $\mathbf{s}^{\prime \prime}$ of this shell, there are different ways to combine these two nonlinear shell variations in a shell $\mathbf{s}^{\prime \prime \prime}$ (see Fig. 6):

(A) the construction of a single geodesic parallelogram $\mathbf{s}, \mathbf{s}^{\prime}, \mathbf{s}^{\prime \prime}, \mathbf{s}^{\prime \prime \prime}$,

(B) the transport of $\left(\mathbf{s}, \mathbf{s}^{\prime \prime}\right)$ along a discrete geodesic from $\mathbf{s}$ to $\mathbf{s}^{\prime}$ via the above sequence of parallelograms, or

(C) the transport of $\left(\mathbf{s}, \mathbf{s}^{\prime}\right)$ along a discrete geodesic from $\mathbf{s}$ to $\mathbf{s}^{\prime \prime}$ via a sequence of parallelograms.
Due to the holonomy in Riemannian manifolds with nonzero curvature, the results will generally differ (Fig. 7).

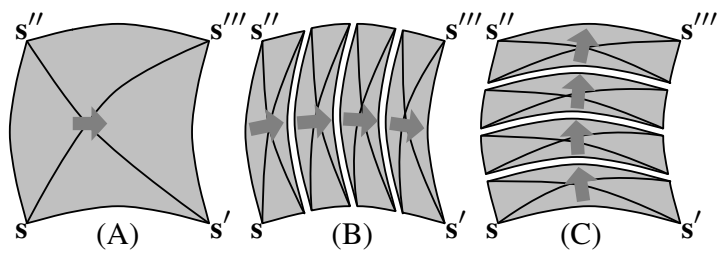

Figure 6: There are different ways of combining two nonlinear shell variations $\mathbf{s}^{\prime}$ and $\mathbf{s}^{\prime \prime}$ of $\mathbf{s}$ to a new shell $\mathbf{s}^{\prime \prime \prime}$ via geodesic parallelogram constructions (gray arrows), which lead to different results due to the holonomy of curved spaces (see Fig. 7).
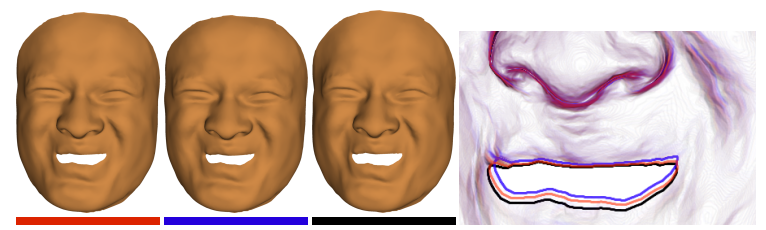

Figure 7: Top: different results of combining an expression of disgust ( $\mathbf{s}^{\prime}$ in Fig. 6) with a smile ( $\left.\mathbf{s}^{\prime \prime}\right)$, starting from a neutral expression (s). Red, blue, and black correspond to (A), $(B)$, and $(C)$ in Fig. 6. (B) is detailed in Fig. 1. The colored contours and shading on the right show differences due to holonomy.

Algorithmic details. The minimization of the discrete path energy for the computation of discrete geodesics is performed by solving the set of Euler-Lagrange equations $\partial_{\mathbf{s}_{k}} \mathbf{E}\left[\mathbf{s}_{0}, \ldots, \mathbf{s}_{K}\right]=0$ for $k=1, \ldots, K-1$ via a Newton iteration with stepsize control. In each Newton step the linear system is solved using an LU factorization. The iteration is stopped if the squared $\ell^{2}$-norm of the Newton step decreases below $3 m(K-1)$ times machine epsilon, where $3 m(K-1)$ is the total number of degrees of freedom. This happens well inside the quadratic convergence regime. To start the Newton iteration within its domain of convergence, we first initialize with a discrete path $\left(\mathbf{s}_{A}, \mathbf{s}_{A}, \ldots, \mathbf{s}_{A}, \mathbf{s}_{B}\right)$ and perform two to three Gauss-Seidel type iterations, in which we alternatingly update all odd and all even shapes. For a very 
small weight of the bending term it is sometimes necessary to first compute a geodesic with a larger weight and then use this as initialization. Geodesic extrapolation is based on the same Newton scheme, this time used to find a root $\mathbf{s}_{2}$ of the nonlinear function $\mathbf{s}_{2} \mapsto \partial_{2} \mathbf{E}\left[\mathbf{s}_{0}, \mathbf{s}_{1}, \mathbf{s}_{2}\right]$. Per shape it typically only needs a handful of steps until convergence and roughly four seconds (serially) for a mesh with 6000 nodes.

\section{The geometry of shell space}

Figures 1 to 4 show applications of geodesic inter- and extrapolation as well as parallel transport. In the following we will briefly discuss the nature of those tools and of the Riemannian setup.

The interplay of physics and geometry. Our metric on the space of shells and our discrete approximation of the path energy are motivated by physics (especially by the notions of elastic energy and viscous dissipation). Note however that our proposed framework for shape exploration is purely geometric, based on the notion of a Riemannian manifold of shells and Riemannian operators such as the exponential or logarithmic map. In particular we do not perform a physical simulation, but instead compute special geometric curves (geodesics) within a geometric space of shells. In the context of shape animation, this framework offers a well-founded alternative to performing physical simulations (which are for instance used in [HSTP11, TSSH13]).

What are the differences? Assume, an animation direction is given as the initial velocity of a shell. In a physical simulation of an elastic shell based on Newton's law of motion, the elastic forces pull the shell back to the original configuration so that the velocity decreases and is finally reversed: the shell swings back and forth, performing a (potentially nonlinear) oscillation around a rest position (see [TSSH13, Fig. 1]). From an energetic viewpoint, the total physical energy is conserved, but it shifts back and forth between potential and kinetic energy. Damping might be added to smooth out irregularities of the initial velocity. In the case of a purely viscous shell there are only damping forces and no elastic forces so that the motion simply decays exponentially. In contrast to those two situations, using our geometric approach of shooting geodesics, the velocity and the energy dissipation rate stay roughly constant (see Fig. 3, 9) so that the initial character of the motion is maintained throughout (as is most plainly visible in Fig. 8).

Despite the distinct differences in the velocity time profiles, the animation paths of physical and geometric computations will be quite similar. Indeed, the elastic model and the Riemannian metric both prefer the same directions: The eigenmodes of the elastic energy Hessian with small eigenvalue correspond to low frequency oscillations with little elastic restoring force so that an initial velocity in such a direction will lead to a large motion amplitude (see [HSTP11, Fig. 1]). Likewise, these same directions are also low order eigenvectors of our metric, meaning that they produce the

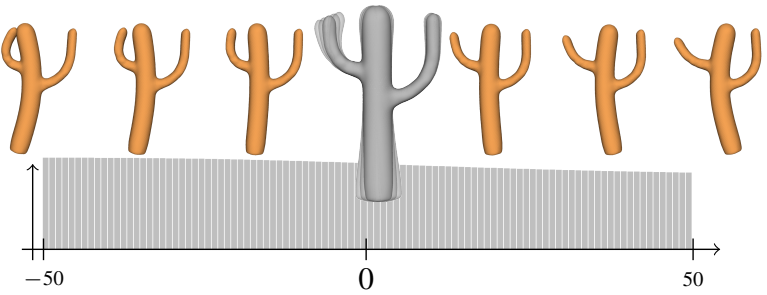

Figure 8: Sample shells from the geodesic extrapolation along an initial displacement given by the fifth non-trivial eigenmode of the elastic Hessian. The diagram shows the dissipation between each two consecutive shapes in the finer resolved computation.

least dissipation for a given magnitude of motion. We refer to Fig. 8 and 9 for a shooting of discrete geodesics in these preferred directions.

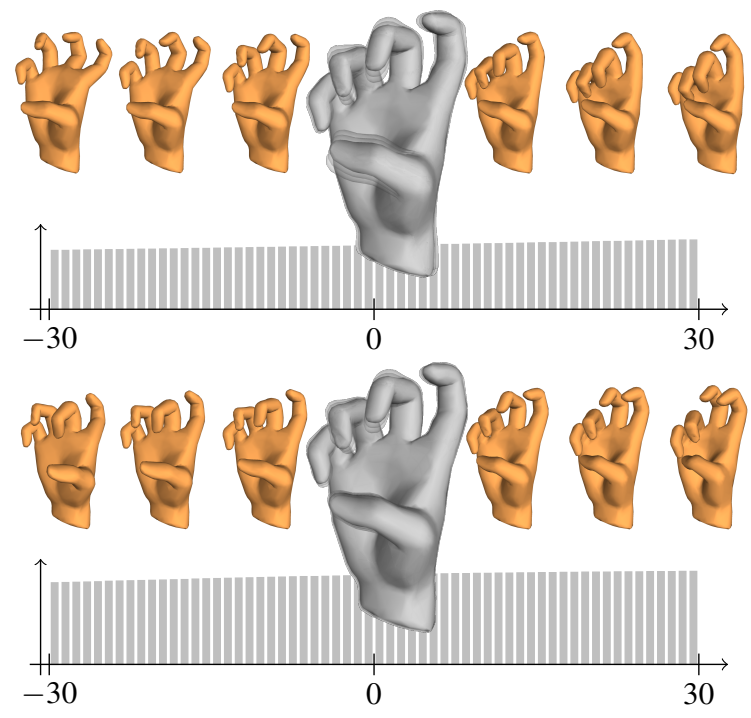

Figure 9: Geodesic extrapolation from a shell along two different initial displacements, given by the second and fourth non-trivial eigenmode of the Hessian of the elastic energy with eigenvalues $\lambda=4.85363$ (top) and $\lambda=5.91444$ (bottom), respectively. Shown are the initial shell with the corresponding eigenmode scaled by \pm 5 and the extrapolated shells based on 10, 20 and 30 iterations of the extrapolation. The diagrams display the almost constant dissipation between each two consecutive shells. (Nominal shell thickness is $\delta=0.032$.)

Shell space exploration. The space of shells is not flat, but curved. So far, we know little about the local and global curvature of shape spaces. Even though such knowledge may not have direct applications in shape animation or exploration, it will help to understand what can be expected from different geometric modeling approaches. For instance, shape spaces of an elliptic character (with positive curvature) 
may exhibit multiple different shortest geodesics between two given shapes. Also, initially parallel geodesics converge, implying less freedom for shape deformation. On the other hand, geodesic paths in shape spaces of hyperbolic nature (with negative curvature) are expected to be unique, however, initially close geodesics diverge exponentially, implying instability with respect to small velocity perturbations. Finally, if a shape space turns out to be almost flat, then after a reparameterization of the shape space geodesics become straight lines and all Riemannian operations become linear, which might be exploited for efficient algorithms.

For shell space, a rough understanding of its curvature has yet to be developed, however, Fig. 10 provides a first glimpse into this direction. Here, a triangle of three geodesics is computed between three given shells $D, E$, and $F$, as well as its midpoint $M$ (the point with the least average geodesic distance to the vertices). The geodesic distances from $M$ to points along the triangle edges are slightly larger than they would be in a flat Riemannian space, indicating a positive sectional curvature. (Similarly, the side lengths are slightly shorter than expected for a flat triangle with the same midpoint-vertex distances.) The curvature is more pronounced for smaller nominal shape thickness $\delta$. Note that $\delta^{2}$ can be interpreted as the relative weight between bending and membrane dissipation. Intuitively, the bending term prefers paths along which the local shell curvature at each point transitions uniformly in time from the initial to the final value. However, since this cannot happen isometrically, the membrane term prevents such a uniform transition, resulting in a more curved shell space. Note that the shell space curvature is not uniform. Indeed, if in Fig. 10 the shell $M$ is replaced by a flat hexagon, the distance relations even indicate a very slight negative curvature (not displayed).

Another indication of nontrivial shell space curvature is the phenomenon of holonomy: If a tangent vector to the space is parallel transported along a closed curve, it will in general not return to its initial direction (see Fig. 7). This poses limitations on the extent to which parallel transport can robustly be used for deformation transfer. However, the example in Fig. 11 indicates that parallel transport still remains a very robust and useful tool. Here, a shape variation is transported along a geodesic triangle, and even though there is a numerical difference between the starting and end point due to holonomy, it is hardly perceptible visually.

\section{Acknowledgements}

We would like to thank Mario Botsch for providing the input meshes for Fig. 2 as well as Tim Winkler, Jens Drieseberg and Kai Hormann for sharing their data and results to enable the comparison in Fig. 4. Facial expression data is taken from [ZSCS04]; we thank William Smith for helping us with processing the data. Additional data was kindly provided by Niloy Mitra and Olga Sorkine. Behrend Heeren was supported by the BMBF via CROPSENSe.net; Martin Rumpf
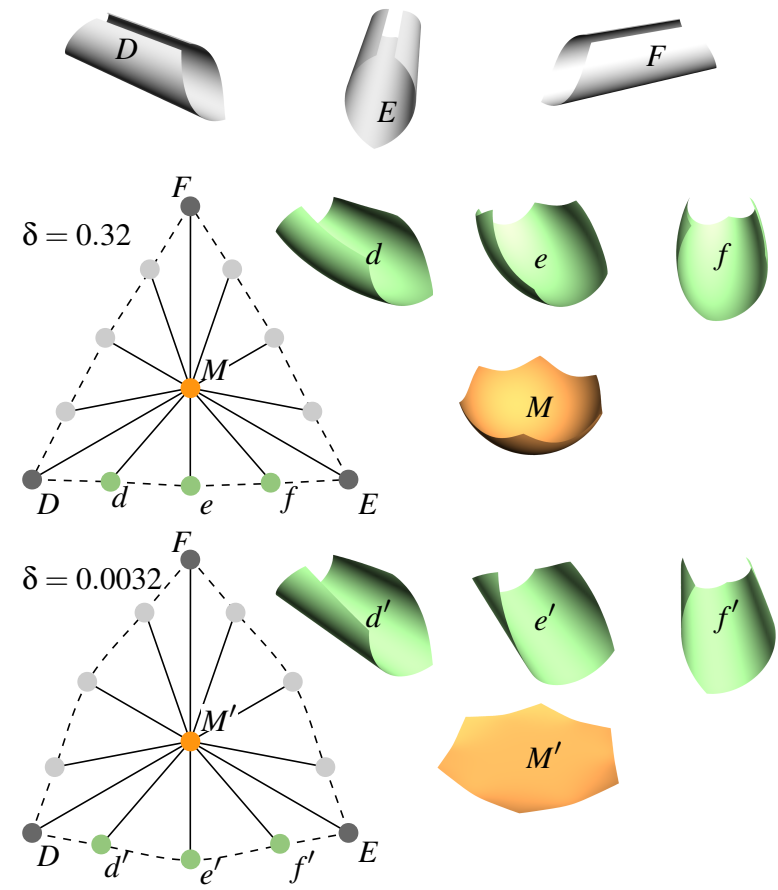

Figure 10: The shell space is curved: The three shapes $D, E, F$ span two different geodesic triangles for nominal shell thickness $\delta=0.32$ and $\delta=0.0032$. These triangles are computed based on discrete geodesics with $K=17$. On the geodesic edge from $D(t=0)$ to $E(t=1)$ intermediate shells are displayed for $t=\frac{1}{4}\left(d, d^{\prime}\right), t=\frac{1}{2}\left(e, e^{\prime}\right)$, and $t=\frac{3}{4}$ $\left(f, f^{\prime}\right)$. Furthermore, the associated geodesic barycenters, $M$ and $M^{\prime}$ respectively, are shown. The points on the edges of the geodesic triangles are positioned such that the length of solid line segments reflects the geodesic distance between the end points. The resulting triangle shapes indicate positive curvature on the space of shells.

was supported by DFG project Ru 567/14-1. Max Wardetzky was partially supported by the BMBF project MuSiKa.

\section{References}

[BHM11] Bauer M., HARMS P., Michor P. W.: Sobolev metrics on shape space of surfaces. J. Geom. Mech. 3, 4 (2011), 389-438. 3

[BMTY02] Beg M. F., Miller M., Trouvé A., Younes L.: Computational anatomy: Computing metrics on anatomical shapes. In Proc. IEEE ISBI (2002), pp. 341-344. 2

[BPGK06] Botsch M., Pauly M., Gross M., Kobbelt L.: PriMo: Coupled prisms for intuitive surface modeling. In Proc. Symp. Geom. Proc. (2006), pp. 11-20. 2

[BS08] Botsch M., SOR KINE O.: On linear variational surface deformation methods. IEEE Trans. Vis. Comp. Graph. 14 (2008), 213-230. 2

[Cia00] Ciarlet P. G.: Mathematical Elasticity, Vol III: Theory of Shells. North-Holland, 2000. 3 


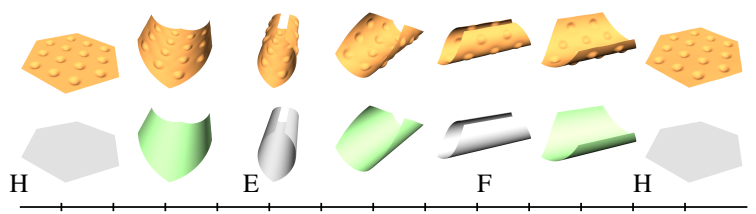

Figure 11: For the geodesic triangle HEF (bottom; intermediate shells in green) with $\delta=0.0032$ and $H$ being the flat hexagon, a bump map is transported along the edges $H \rightarrow E \rightarrow F \rightarrow H$ (top row). Each edge is approximated by a discrete geodesic with $K=4$, and the bump map is scaled by a factor $\varepsilon=10^{-2}$ while being transported. The $L^{2}(H)$ distance between the initial and the final bump map on the shell $H$ due to holonomy is 0.0125 .

[EPS72] Ehlers J., Pirani F. A. E., Schild A.: The geometry of free fall and light propagation. In General relativity. Clarendon Press, Oxford, 1972, pp. 63-84. 5

[FB11] FröHLICH S., Botsch M.: Example-driven deformations based on discrete shells. Comp. Graph. Forum 30, 8 (2011), 2246-2257. 2, 5, 6

[FJSY09] Fuchs M., JÜTtLeR B., ScherZer O., YANG H.: Shape metrics based on elastic deformations. J. Math. Imaging Vis. 35, 1 (2009), 86-102. 2

[FLPJ04] Fletcher P., Lu C., Pizer S., Joshi S.: Principal geodesic analysis for the study of nonlinear statistics of shape. IEEE Trans. Med. Imag. 23, 8 (2004), 995-1005. 2

[GGRZ06] GRInspun E., Gingold Y., Reisman J., Zorin D.: Computing discrete shape operators on general meshes. Comp. Graph. Forum 25, 3 (2006), 547-556. 2

[GHDS03] Grinspun E., Hirani A. N., Desbrun M., SCHRÖDER P.: Discrete shells. In Proc. Symp. Comp. Anim. (2003), pp. 62-67. 2

[HLW06] HaIRer E., Lubich C., WANNER G.: Geometric numerical integration, structure-preserving algorithms for ordinary differential equations, vol. 31 of Comp. Math. Springer, 2006. 3

[HRWW12] HEEREN B., RUMPF M., WARDETZKY M., WiRTH B.: Time-discrete geodesics in the space of shells. Comp. Graph. Forum 31, 5 (2012), 1755-1764. 2, 3, 4

[HSTP11] Hildebrandt K., Schulz C., Tycowicz C. v., POLTHIER K.: Interactive surface modeling using modal analysis. ACM Trans. Graph. 30, 5 (2011), 119:1-11. 2, 7

[Hug87] Hughes T. J. R.: Finite Element Method - Linear Static and Dynamic Finite Element Analysis. Prentice-Hall, 1987. 2, 4

[JZLG09] Jin M., ZenG W., LuO F., Gu X.: Computing Teichmüller shape space. IEEE Trans. Vis. Comp. Graph. 15, 3 (2009), 504-517. 3

[KKG*12] Kurtek S., Klassen E., Gore J., Ding Z., SRIVASTAVA A.: Elastic geodesic paths in shape space of parametrized surfaces. IEEE Trans. Pattern Anal. Mach. Intell. 34, 9 (2012), 1717-1730. 3

[KMN00] Kheyfets A., Miller W. A., Newton G. A.: Schild's ladder parallel transport procedure for an arbitrary connection. Internat. J. Theoret. Phys. 39, 12 (2000), 2891-2898.

[KMP07] Kilian M., Mitra N. J., Pottmann H.: Geometric modeling in shape space. In ACM Tran. Graph. (2007), vol. 26, pp. $1-8.2,5$
[LMOW04] Lew A., Marsden M., Oritz M., West M.: Variational time integrators. Int. J. Numer. Meth. Engng. 60 (2004), 153-212. 3

[Mil63] MILnOR J.: Morse Theory. Princeton University Press, 1963. 3

[MM06] MichOR P. W., MUMFORD D.: Riemannian geometries on spaces of plane curves. J. Eur. Math. Soc. 8 (2006), 1-48. 2

[SCC06] Schmidt F. R., Clausen M., Cremers D.: Shape matching by variational computation of geodesics on a manifold. In Pattern Recognition (2006), vol. 4174 of LNCS, Springer, pp. 142-151. 2

[SJJK06] SRivastava A., Jain A., Joshi S., Kaziska D.: Statistical shape models using elastic-string representations. In Asian Conf. Comp. Vision (2006), vol. 3851 of LNCS, Springer, pp. 612-621. 2

[Str45] StRUTT J.: Theory of sound: Vol. 2. Dover Publications, 1945. 4

[SYM07] Sundaramoorthi G., YeZzi A., Mennucci A.: Sobolev active contours. Inter. J. Comp. Vision 73, 3 (2007), 345-366. 2

[TPBF87] Terzopoulos D., Platt J., Barr A., Fleischer K.: Elastically deformable models. In Proc. SIGGRAPH (1987), pp. 205-214. 2

[TSSH13] Tycowicz C. V., Schulz C., Seidel H.-P., HILDEBRANDT K.: An efficient construction of reduced deformable objects. ACM Trans. Graph. 32, 6 (2013). 2, 7

[WBRS11] Wirth B., BAR L., RUMPF M., SAPIRo G.: A continuum mechanical approach to geodesics in shape space. Inter. J. Comp. Vision 93, 3 (2011), 293-318. 2

[WDAH10] Winkler T., Drieseberg J., Alexa M., HorMANN K.: Multi-scale geometry interpolation. Comp. Graph. Forum 29, 2 (2010), 309-318. 2, 6

[You10] Younes L.: Shapes and diffeomorphisms, vol. 171 of Appl. Math. Sciences. Springer, 2010. 2

[ZSCS04] Zhang L., SNavely N., Curless B., Seitz S. M.: Spacetime faces: High resolution capture for modeling and animation. ACM Tran. Graph. 23, 3 (2004), 548-558. 8

[ZT00] ZIENKIEWICZ O. C., TAYLOR R. L.: The finite element method: The basis, 5th ed., vol. 1. Butterworth and Heinemann, 2000. 2,4

\section{Appendix}

Proof of Theorem 1. Let $s \in \mathcal{S}$ be a surface embedded into $\mathbb{R}^{3}$. Since the energy $\mathcal{W}$ is non-negative and minimized by the identity deformation (conditions (i), (ii), (iii)), it follows that $g_{s}=\operatorname{Hess}(\mathcal{W})$ is positive semi-definite. It remains to show that $g_{s}$ is definite on the complement of rigid transformations of $s$. By positive semi-definitness, $\operatorname{Hess}(\mathcal{W})(v, v)=0$ if and only if $\operatorname{Hess}\left(\mathcal{W}_{\text {mem }}\right)(v, v)=0$ and $\operatorname{Hess}\left(\mathcal{W}_{\text {bend }}\right)(v, v)=0$. Hence it suffices to study the null spaces of $\operatorname{Hess}\left(\mathcal{W}_{\text {mem }}\right)$ and $\operatorname{Hess}\left(\mathcal{W}_{\text {bend }}\right)$. To simplify the exposition, we confine ourselves to the case where membrane and bending energy densities are given by the squared Frobenius norm. The general case works completely analogous.

In order to study variations, we consider a smooth 1parameter family $\left(\phi_{t}\right)$ of diffeomorphisms of $\mathbb{R}^{3}$ with $\phi_{0}=\mathbb{1}$. 
To abbreviate notation, let $\mathrm{I}_{t}$ and $\mathrm{II}_{t}$ denote the first and second fundamental forms induced by $\phi_{t}$ and pulled back to $s$, respectively, i.e., ,

$$
\begin{aligned}
\mathrm{I}_{t}(v, w) & =\mathrm{I}_{\phi_{t}(s)}\left(d \phi_{t}(v), d \phi_{t}(w)\right) \\
\mathrm{II}_{t}(v, w) & =\mathrm{II}_{\phi_{t}(s)}\left(d \phi_{t}(v), d \phi_{t}(w)\right)
\end{aligned}
$$

for vectors $v$ and $w$ tangent to $s$. In what follows, a superscript dot denotes differentiation with respect to the $t$ variable at the point $t=0$.

Lemma $1 \operatorname{Hess}\left(\mathcal{W}_{\text {mem }}\right)(\dot{\phi}, \dot{\phi})=0$ iff $\phi_{t}$ induces an infinitesimally isometric deformation of the surface $s$ (iff $\left.\dot{\mathrm{I}}\right|_{t=0}=0$ ).

Proof Let $\mathrm{I}=\mathrm{I}_{0}$. Then at $t=0$ we get

$$
\begin{aligned}
& \text { Hess } \mathcal{W}_{\text {mem }}(\dot{\phi}, \dot{\phi})=\left.\frac{d^{2} \mathcal{W}_{\text {mem }}\left[\phi_{t}\right]}{d t^{2}}\right|_{t=0}=\left.\frac{d^{2}}{d t^{2}} \int_{s}\left\|\mathrm{I}-\mathrm{I}_{t}\right\|^{2} d s\right|_{t=0} \\
& =\left.2 \frac{d}{d t} \int_{s}\left\langle\mathrm{I}_{t}-\mathrm{I}, \dot{\mathrm{I}}_{t}\right\rangle d s\right|_{t=0}=\left.2 \int_{s}\left\|\dot{\mathrm{I}}_{t}\right\|^{2}\right|_{t=0} d s .
\end{aligned}
$$

The last term vanishes iff $\dot{\mathrm{I}}=0$ a.e. (everywhere due to smoothness).

In order to investigate $\operatorname{Hess}\left(\mathcal{W}_{\text {bend }}\right)$, we first require a well-known fact of how to represent the second fundamental form of a surface immersed into $\mathbb{R}^{3}$. Let $v$ and $w$ be two tangential vector fields to $s$, and let hess $s_{s}$ be the Hessian operator on the surface $s$ induced by the first fundamental form on $s$-this Hessian is not to be confused with the Hessian Hess on shell space. Then $\operatorname{II}(v, w)=\operatorname{hess}_{s}\left(\phi_{0}\right)(v, w) \cdot n$. or in short II $=\operatorname{hess}_{s}(x) \cdot n$, where $\phi_{0}: s \rightarrow \mathbb{R}^{3}$ is the embedding and $n$ is the unit normal vector field of $s$, respectively. Here hess $_{s}$ acts separately on each of the three components of $\phi_{0}$. In particular, notice that hess $s_{s}\left(\phi_{0}\right)(v, w)$ takes values in the normal bundle of $s$.

Lemma 2 If the deformation $\phi_{t}$ induces an infinitesimally isometric deformation of $s$, i.e., , if $\dot{\mathrm{I}}=0$, then

$$
\operatorname{Hess}\left(\mathcal{W}_{\text {bend }}\right)(\dot{\phi}, \dot{\phi})=2 \int_{s}\left\|\operatorname{hess}_{s}(\dot{\phi}) \cdot n\right\|^{2} d s
$$

where $n$ denotes the unit normal vector field on $s$.

Proof We abbreviate notation by referring to $n_{t}$ and $H_{t}$ as the unit normal field and the Hessian operator of the surface $\phi_{t}(s)$ pulled back to $s$, respectively, i.e., , $n_{t}=n_{\phi_{t}(s)} \circ \phi_{t}$ and for a function $f: s \rightarrow \mathbb{R}$ we have

$$
H_{t}(f)(v, w)=\operatorname{hess}_{\phi_{t}(s)}\left(f \circ \phi_{t}^{-1}\right)\left(d \phi_{t}(v), d \phi_{t}(w)\right),
$$

where $v$ and $w$ are tangent to $s$. Then it follows from the previous discussion that $\mathrm{II}_{t}=H_{t}\left(\phi_{t}\right) \cdot n_{t}$. Thus at $t=0$ we have

$$
\begin{aligned}
& \operatorname{Hess}\left(\mathcal{W}_{\text {bend }}\right)(\dot{\phi}, \dot{\phi})=\frac{d^{2}}{d t^{2}} \mathcal{W}_{\text {bend }}\left[\phi_{t}\right] \\
& =\frac{d^{2}}{d t^{2}} \int_{s}\left\|H_{t}\left(\phi_{t}\right) \cdot n_{t}-\operatorname{hess}_{s}\left(\phi_{0}\right) \cdot n\right\|^{2} d s \\
& =2 \int_{s}\left\|\frac{d}{d t}\left(H_{t}\left(\phi_{t}\right) \cdot n_{t}\right)\right\|^{2} d s .
\end{aligned}
$$

Expanding gives

$$
\frac{d\left(H_{t}\left(\phi_{t}\right) \cdot n_{t}\right)}{d t}=\frac{d H_{t}\left(\phi_{0}\right)}{d t} \cdot n+\operatorname{hess}_{s}(\dot{\phi}) \cdot n+\operatorname{hess}_{s}\left(\phi_{0}\right) \cdot \dot{n} .
$$

To prove the claim, it suffices to show that both $\frac{d}{d t}\left(H_{t}\right)$ and $\operatorname{hess}_{s}\left(\phi_{0}\right) \cdot \dot{n}$ vanish at $t=0$. To see that the first term vanishes, recall that the Hessian of a smooth function $f$ on a Riemannian manifold can be expressed in a local coordinate chart $\left(q^{1}, q^{2}\right)$ as

$$
\operatorname{hess}_{s}(f)=\nabla d f=\sum_{i, j, k}\left(\frac{\partial^{2} f}{\partial q^{i} \partial q^{j}}-\frac{\partial f}{\partial q^{k}} \Gamma_{i j}^{k}\right) d q^{i} \otimes d q^{j},
$$

where the Chistoffel symbols of the Levi-Civita connection are given by $\Gamma_{i j}^{k}=\frac{1}{2} g^{k l}\left(g_{i l, j}+g_{j l, i}-g_{i j, l}\right)$. By assumption we have $\dot{\mathrm{I}}=0$. Hence the Riemannian metrics $g_{t}=\mathrm{I}_{t}$ satisfy $\dot{g}_{i j}=\dot{g}^{k l}=0$ and hence $\dot{\Gamma}_{i j}^{k}=0$. Hence $\frac{d}{d t}\left(H_{t}\right)=0$ at $t=0$. It remains to show that hess ${ }_{s}\left(\phi_{0}\right) \cdot \dot{n}=0$, which immediately follows from the fact that hess $s_{s}\left(\phi_{0}\right)$ takes values in the normal bundle of $s$ and from $\dot{n} \cdot n=0$.

We are now in the position to prove our theorem.

Proof [of Theorem 1] If the deformations $\phi_{t}$ are such that $\dot{\phi}$ is in the kernel of $\operatorname{Hess}(\mathcal{W})$, then $\dot{\phi}$ is in the kernel of both $\operatorname{Hess}\left(\mathcal{W}_{\text {mem }}\right)$ and Hess $\left(\mathcal{W}_{\text {bend }}\right)$. By Lemma $1, \dot{\phi}$ then induces an infinitesimal isometry, i.e., , $d \dot{\phi}$ is an infinitesimal rotation for every point on $s$. Such an infinitesimal rotation can at each point be represented by a skew-symmetric matrix or equivalently the cross product with a vector $a \in \mathbb{R}^{3}$ parallel to the rotation axis. Now let $x: s \rightarrow \mathbb{R}^{3}$ denote the restriction of $\phi_{0}=\mathbb{1}$ to $s$. Since $d x$ acts as the identity on tangent vectors and $d \dot{\phi}$ is an infinitesimal rotation, there exists a (unique) $a: s \rightarrow \mathbb{R}^{3}$ such that $d \dot{\phi}=a \times d x$. By Lemma 2, we additionally have that hess $s_{s}(\dot{\phi}) \cdot n=0$. Since for any (smooth) $f: s \rightarrow \mathbb{R}$ one has hess $(f)=\nabla d f$, where $\nabla$ denotes covariant differentiation, we obtain

$$
\begin{aligned}
0 & =(\nabla d \dot{\phi}) \cdot n=\nabla(a \times d x) \cdot n \\
& =(\nabla a \times d x+a \times \nabla d x) \cdot n=(\nabla a \times d x) \cdot n,
\end{aligned}
$$

where the last equality follows from the fact that $\nabla d x=$ $\operatorname{hess}_{s} x$ takes values in the normal bundle of $s$. Additionally, observe that the symmetry of Hessians and the identity

$$
\operatorname{hess}_{s}(\dot{\phi})=\nabla d \dot{\phi}=\nabla a \times d x+a \times \operatorname{hess}_{s}(x)
$$

implies that $(\nabla a \times d x)$ is symmetric, i.e., , $\nabla_{v} a \times w=$ $\nabla_{w} a \times v$ for all tangent vector fields $v, w$. In particular, fix a tangential field $v$ that does not vanish in some open subset $U \subset s$ and (locally) choose $w$ such that $v \times w=n$ on $U$. Then

$$
0=\left(\nabla_{v} a \times w\right) \cdot w=\left(\nabla_{w} a \times v\right) \cdot w=n \cdot \nabla_{w} a
$$

on $U$. Therefore, since $U$ and $v$ are arbitrary, $\nabla_{w} a$ is a tangential field for all tangent vector fields $w$; hence, $\nabla a \times d x$ takes values in the normal bundle, and from $(\nabla a \times d x) \cdot n=0$ we deduce that $\nabla a=0$. Hence $a$ is constant and therefore $\dot{\phi}=a \times x+b$ for some constant $b \in \mathbb{R}^{3}$ is an infinitesimal rigid body motion in $\mathbb{R}^{3}$, which proves the claim. 